\title{
Las Unidades de Policía frente a la Violencia de GÉNERo, en Grandes Municipios Rurales
}

\author{
Antonio Jesús Yugueros García

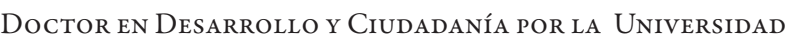 \\ Pablo de Olavide$$
\text { ○滋亏 }
$$

\begin{abstract}
RESUMEN
La violencia contra las mujeres ha pasado de ser un problema del ámbito privado, familiar, a constituir una problemática de dominio público, de gran transcendencia porque afecta a los derechos humanos de muchas mujeres a nivel mundial, máxime si esta violencia es ejercida por la pareja o expareja, que lo hace más execrable. Los estados democráticos, tienen como principal misión, velar por la vida e integridad física de su ciudadanía, y por ende luchar contra esta lacra social, por este motivo las Unidades de Policía de estos países, como servidores públicos de sus conciudadanos, tienen el reto de implementar las medidas necesarias al fin enunciado.
\end{abstract}

Pala bras Clave: Violencia de Género, Policía, Seguridad ciudadana, pareja.

\section{INTRODUCCIÓN TEÓRICA}

\subsection{Violencia de GÉNERo}

La Declaración sobre la Eliminación de la Violencia contra la Mujer, aprobada en Resolución de la Asamblea General de Naciones Unidas 48/104, del 20 de diciembre de 1993 (ONU, 1993), es el primer documento a nivel internacional, que aborda de manera clara y específica esta tipología de violencia, definiendo que la violencia contra la mujer es todo acto de violencia basado en la pertenencia al sexo femenino, que tenga o pueda tener como resultado un daño o sufrimiento físico, sexual o psicológico para la mujer, así como las amenazas de tales actos, la coacción o la privación arbitraria de la libertad, tanto si se produce en la vida pública como en la vida privada.

Los actos que constituyen violencia contra las mujeres, aunque no puedan limitarse a estos solamente, son: 
- La violencia física, sexual y psicológica que se produce en la familia, incluidos los malos tratos, el abuso sexual de las niñas en el hogar, la violencia relacionada con la dote, la violencia por el marido, la mutilación genital femenina y otras prácticas tradicionales nocivas para la mujer, los actos de violencia perpetrados por otros miembros de la familia y la violencia relacionada con la explotación.

- La violencia física, sexual y psicológica perpetrada dentro de la comunidad en general, inclusive la violación, el abuso sexual, el acoso y la intimidación sexual en el trabajo, en instituciones educacionales y en otros lugares, la trata de mujeres y la prostitución forzada.

- La violencia física, sexual y psicológica perpetrada o tolerada por el Estado, dondequiera que ocurra.

\subsection{La Violencia de Género en las relaciones de Pareja}

Por otra parte, la LO.1/2004, de 28 de diciembre, de Medidas de Protección Integral contra la Violencia de Género (LO 1/2004), enuncia que esta violencia es una manifestación de la discriminación y situación de desigualdad en las relaciones de poder de los hombres sobre las mujeres, en las relaciones de pareja o expareja.

En este caso se reduce la denominación de violencia de género, a los ilícitos penales cometidos por los hombres pareja o expareja de la mujer víctima, en contraposición a lo enunciado en la Declaración Universal 48/104 antes aludida, que preceptúa la violencia de género con una mayor amplitud de casos de los que pueden ser objeto las mujeres, no haciendo distinciones ni de personas, ni de lugares donde estas agresiones se pueden cometer.

La violencia de género en las relaciones de pareja, han formado parte de la vida cotidiana de las mujeres a lo largo de los tiempos, estaba naturalizada, silenciada, lo que la hacía invisible (Nogueras, 2006; Melero, 2010), con lo cual no estaba reconocida socialmente. Nadie la veía ni la nombraba, incluso las mismas víctimas lo consideraban un asunto de dominio privado; aunque en la actualidad se ha avanzado bastante en la sensibilización ante esta problemática social, todavía existe una actitud silenciosa ante los casos que se dan habitualmente en nuestra sociedad. Gracias a la denodada lucha de los movimientos de mujeres, se han podido visibilizar estos hechos, haciendo que pasen a formar parte del ámbito público, instando ante los 
poderes públicos a que se promulgaran leyes encaminadas a la eliminación de este tipo de violencia, y a que se implementaran medidas de protección integral a las mujeres víctimas de esta violencia patriarcal.

\subsection{Las Fuerzas de seguridad ante la Violencia de GÉNERO}

Los Puestos de la Guardia Civil, en las poblaciones rurales, son los organismos donde suele atenderse por primera a vez, a las mujeres víctimas de violencia de género en las relaciones de pareja o expareja, bien por ser el lugar más próximo a sus domicilios, donde pueden acudir a solicitar auxilio urgente, al peligrar sus vidas, integridad física o la de sus hijos e hijas, para denunciar los hechos de los que están siendo objeto con el propósito de ponerles fin, o requerir información sobre los derechos que les asisten.

Las Fuerzas y Cuerpos de Seguridad, deberán tener en cuenta en sus actuaciones lo preceptuado en el Protocolo de Actuación de las Fuerzas y Cuerpos de Seguridad, y de Coordinación con los Órganos Judiciales para la Protección de las Víctimas de Violencia Doméstica y de Género (Protocolo, 2004).

\subsubsection{CONOCIMIENTO DE LOS HeChOS}

La forma más habitual de conocer los casos de violencia de género, es por personación de las mujeres víctimas en los Puestos de la Guardia Civil, para interponer la oportuna denuncia. Este paso es trascendental para ellas, con lo cual se les debe tratar con la mayor profesionalidad y sensibilidad posible.

Otra forma habitual de conocer casos de violencia contra las mujeres es a través de la derivación que realizan los Servicios de Atención a la Mujer, o Asociaciones de Mujeres, por entender necesitan protección inmediata para ella o para sus hijos e hijas.

\subsubsection{Personación y PRIMERa atención}

Cuando la ciudadanía se persona en un acuartelamiento de la Guardia Civil, para interponer denuncia, son atendidos en primera instancia 
por el denominado Guardia de Puertas, que es el/a agente encargado/a de recibir a las personas que llegan a las dependencias policiales, quien lo derivará al servicio correspondiente.

En el caso de las mujeres víctimas de violencia de género, serán dirigidas a las dependencias de violencia de género en los Puestos principales, o al personal encargado de este cometido en los demás Puestos ordinarios, donde serán escuchadas activamente e informadas de sus derechos.

\subsubsection{RECEPCIÓN DE LA DENUNCIA}

Para las mujeres víctimas de violencia de género en las relaciones de pareja o expareja, acudir a los servicios policiales a interponer una denuncia, no es fácil, de suerte que habrá que aprovechar esta circunstancia para brindarle todo el apoyo que necesiten al objeto de que puedan salir de la situación en la que se encuentran (Garrido, 2011).

En el acto de la recepción de la denuncia, se les dispensará el tiempo que necesiten para relatar lo que les ha sucedido, espacios para que expresen sus sentimientos, posiblemente sea la primera vez que se desahogan al relatar los hechos que les están ocurriendo.

Si los malos tratos que denuncia son psicológicos, sería conveniente, remontar la historia de vida a cuando comenzaron éstos, que con toda probabilidad se retrotraerán a la época de su noviazgo o inicio de la relación de convivencia. Es importante que queden reflejados en esta diligencia los pormenores que relaten sobre las agresiones sufridas, tanto físicas como psíquicas y/o sexuales, amenazas, menosprecios, insultos; anotando literalmente estas circunstancias, por muy cruentas que parezcan.

\subsubsection{Seguimiento de los Casos y PROTECCión}

La Guardia Civil cuenta con agentes especializados para el tratamiento de los casos de violencia de género en las relaciones de pareja, que se encargan de la atención, recepción de las denuncias y seguimiento de las mujeres víctimas, en los Puestos Principales y en los Puestos ordinarios, estas tareas son realizadas por el conjunto de los componentes de los mismos. 
El seguimiento de las mujeres víctimas de violencia de género en las relaciones de pareja o expareja, comienza con la primera valoración tras la interposición de la denuncia, denominada Valoración Policial del Riesgo (VPR), que está reglamentada por la Instrucción número 10/2007, de la Secretaría de Estado de Seguridad, por la que se aprueba el protocolo para la valoración policial del nivel de riesgo de violencia contra la mujer (Instrucción, 10/2007), modificada y ampliada por las Instrucciones de la misma Secretaría números 14/2007 (Instrucción, 14/2007) y 5/2008 (Instrucción 5/2008).

Esta valoración policial de riesgo se realiza, cumplimentando un formulario informático, instalados en los aplicativos de intranet de los terminales informáticos existentes en las dependencias de los Cuerpos y Fuerzas de Seguridad del Estado. Se realizará por el Instructor de las diligencias policiales, con los datos aportados por la víctima, autor, testigos e informe técnico, y cuyo resultado lo plasmará en el atestado que redacte.

El sistema asignará uno de los siguientes resultados:

- No apreciado.

- Bajo.

- Medio

- Alto

- Extremo.

Dependiendo del resultado obtenido, que será comunicado a la mujer víctima, se adoptarán las siguientes medidas de protección:

\section{Nivel No APRECIADO}

- No se tomarán medidas especiales, solamente las de tipo operativo y asistencial, información de derechos y recursos que tiene a su disposición.

\section{Nivel Bajo}

\section{Obligatorias}


- Facilitar a la víctima números de teléfono de contacto permanente (24 horas) con las Fuerzas y Cuerpos de Seguridad más próximas.

- Contactos telefónicos esporádicos con las víctimas.

- Comunicar al agresor que la víctima dispone de un servicio policial de protección.

- Recomendaciones de autoprotección y funcionamiento del servicio de teleasistencia móvil.

\section{Complementarias}

- Contactos esporádicos y discretos con las víctimas (acordar con ella la conveniencia de acudir o no de uniforme, o con vehículo camuflado).

- Confección de una ficha con los datos de la víctima y del agresor, que llevará el personal de patrulla.

- Acompañamiento al denunciado a recoger enseres en el domicilio, si la Autoridad Judicial acuerda su salida del mismo.

\section{Nivel MEDio}

\section{Obligatorias}

- Vigilancia ocasional y aleatoria en domicilio y lugar de trabajo de la víctima, como de los centros escolares de los hijos.

- Acompañamiento a la víctima en actuaciones de carácter judicial o asistencial cuando se considere puede haber peligro para ella.

- Facilitar que se le provea de servicio de teleasistencia móvil.

- Entrevista personal de la víctima con el agente responsable de su protección

\section{Complementarias}

- Comprobar que el agresor cumple con las medidas judiciales de protección.

- Entrevistas con Servicios de Atención a la Mujer, para definir diferentes puntos de protección. 
- Traslado de la víctima pata ingreso en centro de acogida.

\section{Nivel DE Riesgo ALTO}

\section{Obligatorias}

- Vigilancia frecuente y aleatoria en el domicilio y puesto de trabajo de la víctima, así como de los centros educativos de los hijos e hijas.

- Comunicar a la víctima la conveniencia de trasladarse en unos días a un centro de acogida, o al domicilio de un familiar, si todavía no se ha procedido a la detención del autor/maltratador.

- Control esporádico de los movimientos del agresor.

\section{Complementarias}

- Contactos esporádicos con personas del entorno del agresor, de la víctima, vecinos, familia, trabajo, lugares de ocio.

- Procurar que se adopten dispositivos electrónicos de vigilancia con el agresor.

\section{Nivel De Riesgo EXTREMo}

\section{Obligatorias}

- Vigilancia permanente de la víctima, hasta que cesen las circunstancias que permitieron esta medida.

- Control intensivo de la movilidad del agresor, hasta que desaparezca la amenaza sobre la víctima.

- Vigilancia de la entrada y salida de los centros docentes de los hijos e hijas.

La evaluación de riesgo se mantiene actualizada, de tal manera se cumplimentará el formulario denominado Valoración Policial de la Evaluación de Riesgo (VPER), establecidas en los siguientes períodos:

- Nivel extremo: cada 72 horas.

- Nivel alto; cada 7 días. 
- Nivel medio: cada 30 días.

- Nivel bajo: Cada 60 días

También se llevarán a cabo, si lo ordena la Autoridad Judicial, el Ministerio Fiscal o cuando se conozcan cambios significativos en la víctima o en el agresor.

Posteriormente, si la Autoridad Judicial dicta Orden de protección, se tendrá en cuenta lo que se disponga en la misma, que por regla general, en lo concerniente a la adopción de las medidas de protección, serán las de prohibición de acercamiento a la mujer víctima y a su entorno: domicilio, trabajo, etc., y/o prohibición de comunicación por cualquier medio. A partir de este momento, las víctimas pasarán a ser protegidas personalmente, por los agentes del área de violencia de género de los Puestos de la Guardia Civil, donde los hubiere, y en los demás Puestos, por los efectivos del mismo, coordinados por su Comandante de Puesto.

En ambos casos será imprescindible la colaboración de las Unidades que fueran necesarias, para adoptar las medidas resultantes de la VPR, en los casos de mayor riesgo, cuestión que concierne al Mando Operativo de la demarcación territorial de la Guardia Civil, que será quien disponga los efectivos necesarios para consolidar este servicio.

De los cambios que se susciten se dará cuenta a la Autoridad Judicial, y a los servicios oportunos para implementar, en su caso, las medidas de protección adecuadas a la nueva situación.

Otro elemento de protección destinado a las mujeres víctimas de violencia de género en las relaciones de pareja, es el Servicio Telefónico de Atención y Protección para víctimas de violencia de género -ATENPRO-, dirigido por la Delegación del Gobierno para la Violencia de Género, desde enero de 2010, que reemplazó al Servicio de Teleasistencia móvil, referenciado anteriormente en varias ocasiones, que venía realizando este cometido en el que cooperaban el IMSERSO y la FEMP. Este nuevo servicio de asistencia de telefonía móvil, dotado de elementos de localización GPS, permite a las mujeres víctimas contactar con el Centro de Atención a las Mujeres Víctimas, que se encuentra en funcionamiento permanentemente. Solamente oprimiendo un botón tienen acceso al centro de asistencia (ATENPRO, 2013). 
También existe un sistema de seguimiento por medios telemáticos del cumplimiento de medidas cautelares de alejamiento en materia de violencia de género. El inculpado está equipado con un transmisor con una unidad de rastreo GPS, fijado bien a su muñeca o al tobillo. También, a la víctima, se le equipa con un dispositivo GPS, que rastrea constantemente movimientos y ubicación, con tecnología GPS. En una situación de emergencia la víctima puede utilizar el botón establecido al efecto, para efectuar una llamada saliente a un número predeterminado de emergencia (Resolución, 2009).

Igualmente WRAP, que es la web de recursos de apoyo y prevención ante los casos de violencia de género, que se encuentra en la página web del Ministerio de Sanidad, Servicios Sociales e Igualdad, a través de este canal se pueden consultar desde mapas activos los distintos servicios asistenciales en todo el ámbito nacional (Zurita, 2013).

\section{Metodologia}

Para responder al objetivo de esta investigación, se ha efectuado un planteamiento metodológico cuantitativo, para ello se han utilizado cuestionarios estandarizados de preguntas, cerradas, administrados a las mujeres víctimas de violencia de género en las relaciones de pareja o expareja, de la localidad objeto de estudio.

Los cuestionarios han sido administrados por el Punto de Atención a la Mujer de la localidad de estudio, siendo autocumplimentados por las interesadas, que previamente han sido informadas de la investigación que se llevaba a cabo.

El cuestionario consta de una batería de preguntas referidas a aspectos sobre el grado de atención recibido por parte de la Guardia Civil cuando han sido atendidas en los diferentes estadios del proceso por el que han tenido que pasar.

\subsection{Hipotésis y Objetivo}

Se tiene la certeza, que el Cuerpo de la Guardia Civil, realiza una labor positiva en el ámbito de la violencia contra las mujeres en las relaciones de pareja o expareja, aseveración constatada en diversos ámbitos de la sociedad, 
por ello, se hace necesario comprobar si este hipótesis es real o pertenece al ideario social colectivo. El principal objetivo estribará en comprobar la opinión de las mujeres víctimas de esta problemática social, de un municipio perteneciente a la Comunidad Autónoma de Andalucía (España), con más de 25.000 habitantes, que posee Puesto de la Guardia Civil, Cuerpo de seguridad pública, de ámbito nacional, que desarrolla sus funciones en las poblaciones rurales.

\section{Muestra}

La población a estudiar: mujeres víctimas de violencia de género en las relaciones de pareja o expareja que tuvieran orden de protección en vigor. La muestra se ha obtenido de una localidad que cuenta con más de 25.000 habitantes, perteneciente a la Comunidad Autónoma de Andalucía (España). En este municipio existen 100 mujeres con orden de protección. Se ha logrado administrar un máximo de $76(\mathrm{n}=76)$ cuestionarios, lo que supone un $76 \%$. No se ha podido agotar la población completa por problemas de diversa índole, pero, no obstante, se ha logrado una muestra bastante considerable, que permite el estudio pretendido. Esta muestra es viablemente extrapolable a localidades de similares características a la estudiada, siendo su denominador común una población de un número considerable de habitantes.

Gráfico 1. Distribución de la muestra de mujeres en el municipio objeto de estudio

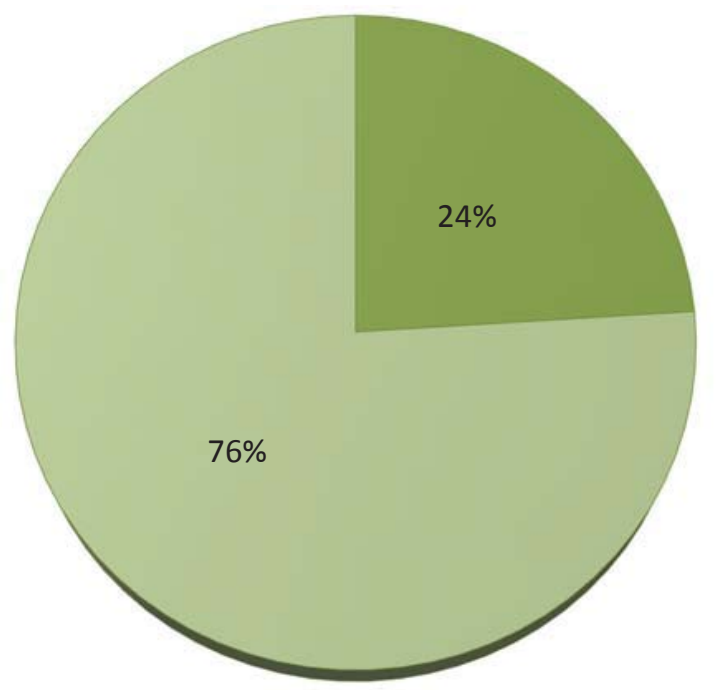

Fuente: elaboración propia 2013

Se ha encuestado al $(76 \%, \mathrm{n}=76)$ de la población, ésta está compuesta por $(\mathrm{n}=100)$ mujeres. 


\section{Atención Recibida por Parte de la Guardia Civil}

\subsection{Primera Atención}

Gráfico 2. Distribución de la variable por grado de satisfacción en la primera atención recibida.

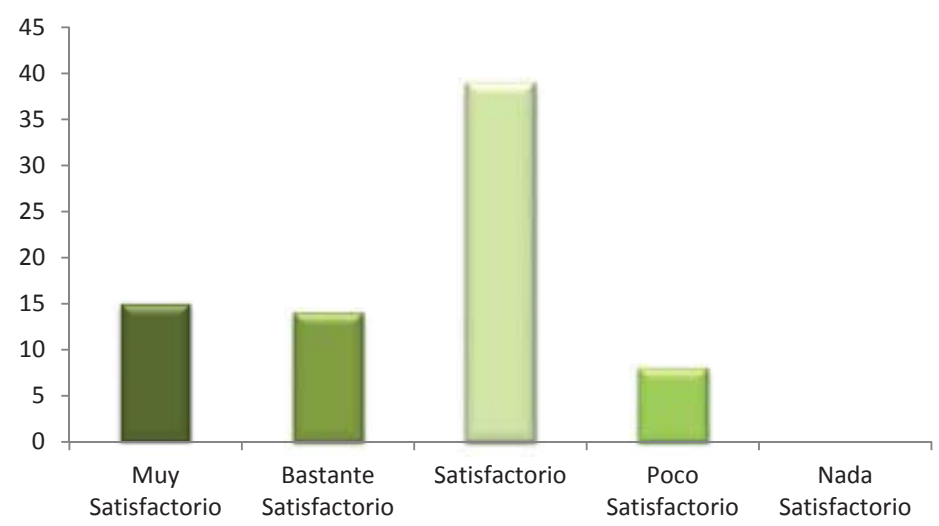

Fuente: elaboración propia 2013

En los datos sobre en la primera atención recibida, han dado los siguientes resultados: muy satisfactoria $19,7 \%(\mathrm{n}=15)$, bastante satisfactoria $18,4 \%(n=14)$, satisfactoria $51,3 \%(n=39)$, poco satisfactoria $10,5 \%(n=8) y$ nada satisfactoria $(\mathrm{n}=0)$.

\subsection{Atención en el ACto de la Recepción de la Denuncia}

Seguidamente se interesa la atención recibida en el acto de recogida de la denuncia, preguntándoles si fueron escuchadas, si pudieron expresar sus sentimientos, les instruyeron de sus derechos, se le facilitó la asistencia letrada, etc.

Gráfico 3. Distribución de la muestra por la valoración en el acto de recepción de la denuncia.

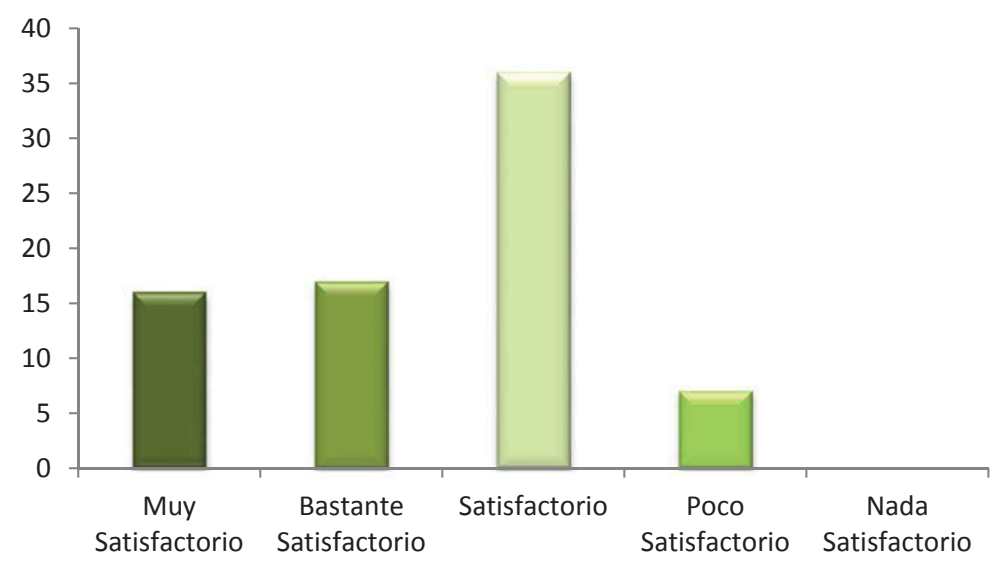

Fuente: elaboración propia 2013 
En esta ocasión se han obtenido los siguientes resultados: muy satisfactoria $21,1 \%(n=16)$, bastante satisfactoria $22,4 \%(n=17)$, satisfactoria $47,4 \%$ (36), poco satisfactoria $9,2 \%(n=7)$ y nada satisfactoria $(n=0)$ (gráfico 18 ).

Sobre la actitud que más sobresalió en los agentes que las atendieron, referido a la atención en el acto de recepción de la denuncia: acogimiento cercano, empatía y profesionalidad. Tenía prisa por terminar. Otra (especificar).

\subsection{Atención en el Seguimiento del Caso}

Gráfico 4. Distribución de la variable por el grado de satisfacción con el seguimiento, localidade A.

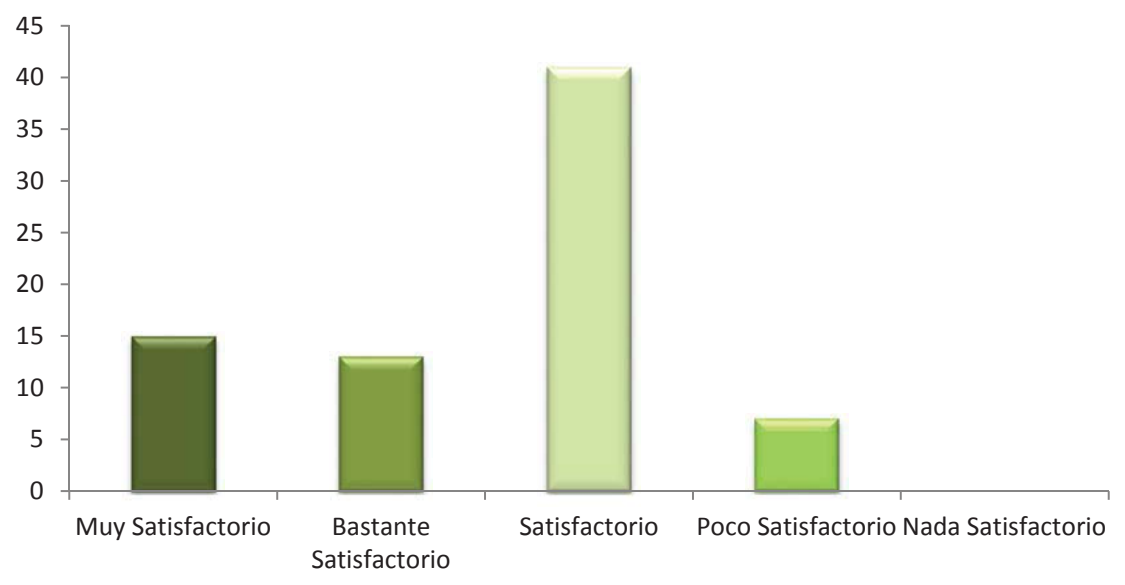

Fuente: elaboración propia 2013

Los resultados obtenidos: muy satisfactoria $19,7 \%(\mathrm{n}=15)$, bastante satisfactoria $17,1 \%(n=13)$, satisfactoria $53,9 \%(41)$, poco satisfactoria $9,2 \%$ $(\mathrm{n}=7)$ y nada satisfactoria $(\mathrm{n}=0)$.

\section{Discusión}

Como ha quedado de manifiesto a lo largo de la investigación realizada, la Guardia Civil lleva a cabo una labor interesante en la atención, protección y seguimiento a las mujeres víctimas de violencia de género en las relaciones de pareja o expareja.

En la hipótesis de partida se aseveraba que el Cuerpo de la Guardia Civil, presta un servicio positivo en este ámbito; para confirmar o refutar ésta, se ha diseñado un objetivo: conocer la opinión de las mujeres víctimas 
de violencia de género, sobre la atención que reciben o han recibido de los agentes de la Guardia Civil, cuando han requerido sus servicios profesionales.

De esta investigación, que se ha focalizado en tres estadios: primera atención, recepción de la denuncia y seguimiento, se desprende lo siguiente:

Se ha podido constatar que en la localidad objeto de estudio, en la primera atención recibida por estas señoras, prevalece el grado de "satisfactoria" con un 51,3\%. Con respecto a la recepción de la denuncia, predomina el porcentaje de atención "satisfactoria": 47,4\%, En el seguimiento de los casos por parte de los agentes encargados de este cometido, ha destacado los resultados de "satisfactoria": 53,9\% y poco satisfactoria: 9,2\%;

Con respecto a los datos negativos sobre el seguimiento, aunque bajo porcentaje, se ha podido investigar, que no se debe a una intervención negativa por parte de la Guardia Civil, es como consecuencia de que la problemática de estas señoras no se ha resuelto debido a múltiples factores, de tal manera que a ellas no se encuentran satisfechas, pero no por falta de atención de parte de los agentes.

De los diversos grupos de mujeres con los que se ha contactado, sobre el servicio prestado por la Guardia Civil en la atención a las mujeres víctimas de violencia en las relaciones de pareja, en todos los casos han respondido, que el trato en general ha sido satisfactorio, y no han sufrido dobles victimizaciones por parte del personal del Cuerpo.

\section{Conclusion}

A modo de conclusión se puede finalizar manifestando, que la Guardia Civil realiza una labor de asistencia social y policial de calidad a las mujeres víctimas de violencia de género en las relaciones de pareja o expareja, como así ha quedado de manifiesto en esta investigación.

Aunque el valor predominante de los resultados es de satisfactoria, si en estos tres estadios se sumaran los datos de muy satisfactoria y bastante satisfactoria, se obtendrían unos datos de calidad extraordinarios, teniendo en cuenta el bajo índice de resultados de poco satisfactoria y nada satisfactoria.

Se entiende que los datos obtenidos en esta investigación, son 
fácilmente extrapolables a poblaciones con igual número de habitantes al estudiado, e igualmente a otros cuerpos policiales con parecidas características al que nos ocupa.

No obstante se sugiere seguir investigando en este campo de la seguridad pública, a fin de lograr una mayor calidad de servicio a las mujeres víctimas de esta lacra social, y conseguir la erradicación de este problema social de nuestros pueblos y ciudades, ya que no se puede entender una sociedad democrática en la que se maltrata a sus mujeres.

Antonio Jesús Yugueros García

Doctor en Desarrollo y CiUdadanía por la Universidad

Pablo de Olavide

\title{
Unidades Policiais contra a Violência de GÊNERO NOS Grandes MunicípIOS RURAIS
}

\begin{abstract}
SUMÁRIO
A violência contra as mulheres deixou de ser um problema de âmbito privado, familiar, e passou a ser um problema de domínio público, de grande importância porque afeta os direitos humanos de muitas mulheres em todo o mundo, especialmente se essa violência é exercida pelo parceiro ou exparceiro, o que torna a mais execrável. Estados democráticos têm como missão principal garantir a vida e a integridade física dos seus cidadãos, e, assim, combater este flagelo social. É por isso que as unidades policiais desses países, como funcionários públicos dos seus concidadãos, são desafiadas para implementar as medidas necessárias com a finalidade enfrentar tal violência.
\end{abstract}

Palavras-Chave: Violência de gênero. Polícia. Segurança pública. Casal.

\section{Police Units against Gender Violence in Great Rural Municipalities}

\begin{abstract}
Violence against women has gone from being a problem of private, of family, to be a problem in the public domain. It is a problem of great importance because it affects the human rights of many women worldwide, especially if the partner or ex-partner exercises the violence, which makes it
\end{abstract}


more execrable. Democratic states have the main mission to ensure the life and physical integrity of its citizens, and consequently combat this social scourge. That is why the police units in these countries, as public servants of their fellow citizens, are challenged to implement the necessary measures to end such violence.

KEYWORDS: Gender Violence. Police. Public Safety. Couple.

\section{BibLiOgRAFía}

ASAMBLEA General de Naciones Unidas (1993). Resolución de la 48/104, del 20 de diciembre. Eliminación de la Violencia contra la Mujer, aprobada.(ONU, 1993).

ATENPRO (2013). "Protocolo de atención del servicio telefónico de atención y protección para las víctimas de la violencia de género". http://www.msssi.gob.es/ssi/violenciaGenero/ Recursos/ ATENPRO/home.htm. (Consultado: 31.01.2016).

GARRIDO, MJ. (2011). Primeros auxilios psicológicos en violencia de género, guía básica. Madrid. Servicio de Psicología de la Guardia Civil.

LEY Orgánica 1/2004, de 28 de diciembre, de Medidas de Protección Integral contra la Violencia de Género (LO. 1/2004). BOE núm. 313, de 29 de diciembre de 2004.

MELERO, N. (2010). Reivindicar la igualdad de mujeres y hombres en la sociedad: una aproximación al concepto de género. Revista Barataria, $n^{\circ} 11$, pp.73-83.

NOGUEIRAS, B. (2006). La violencia en la pareja. En Ruiz-Jarabo, C. y Blanco, P. (Direct.) (2006). La violencia contra las mujeres: Prevención y detección. Editorial Díaz de Santos. España, pp. 39-55.

PROTOCOLO de actuación de las Fuerzas y Cuerpos de Seguridad, y coordinación con los Órganos Judiciales para la protección de las víctimas de violencia doméstica y de género. (Protocolo, 2004).

SECRETARÍA de Estado de Seguridad. (2007). Instrucción número $\mathbf{1 4 / 2 0 0 7}$, de 10 de octubre de 2007, por la que se modifica la Instrucción número 10/2007, de 10 de julio. Madrid, 10 de octubre de 2007. (Instrucción, 14/2007). 
SECRETARÍA de Estado de Seguridad (2008). Instrucción número $\mathbf{5 / 2 0 0 8}$, por la que se modifica la Instrucción 10/2007, de 10 de julio. Madrid, 18 de julio de 2008. (Instrucción, 5/2008).

SECRETARÍA de Estado de Seguridad (2009). Resolución de 17 de ulio de 2009, por la que se acuerda la publicación del Protocolo de actuación para la implantación del sistema de seguimiento por medios telemáticos del cumplimiento de las medidas de alejamiento en materia de Violencia de Género y se ordena la elaboración de las normas técnicas de desarrollo del mismo por los Cuerpos y Fuerzas de Seguridad del Estado. Madrid, 17 de julio de 2009. (Resolución, 2009).

YUGUEROS, AJ. (2013). La intervención de la Guardia Civil, como garante de los Derechos Humanos, en el ámbito de la violencia contra las mujeres, en las relaciones de pareja o expareja. Tesis Doctoral. Universidad Pablo de Olavide (Sevilla). -España-.

ZURITA, J. (2013)."La lucha contra la violencia de género". Revista Seguridad y Ciudadanía, núm. 9, pp. 118-119.

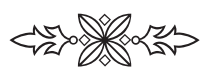

